\title{
Bioanalysis
}

\section{Best practices in performing flow cytometry in a regulated environment: feedback from experience within the European Bioanalysis Forum}

Flow cytometry is a powerful tool that can be used for the support of (pre)clinical studies. Although various white papers are available that describe the set-up and validation of the instrumentation (the flow cytometer) and validation of flow cytometry methods, to date no guidelines exist that address the requirements for performing flow cytometry in a regulated environment. In this manuscript, the European Bioanalysis Forum presents additional practice guidance on the use of flow cytometry in the support of drug development programs and addresses areas that are not covered in the previous publications. The concepts presented here are based on the consensus of discussions in the European Bioanalysis Forum Topic Team 32, in meetings in Barcelona, Limelette and multiple telephone conferences.

First draft submitted: 21 April 2017; Accepted for publication: 5 June 2017;

Published online: 2 August 2017

Keywords: flow cytometry $\bullet$ regulated environment

Flow cytometry is a versatile laser-based platform that has the potential to be used in every stage of a drug or vaccine development program, moving from discovery, through to preclinical and into clinical testing. With the potential for implementation in each of these stages, there is a need to develop robust flow cytometric methods and to validate the methods in alignment with the associated regulatory environments (GLPs, US FDA, EMA, International Organization for Standardization [ISO] and Standard for Clinical Laboratory Accreditation [ISO15189]).

Assays that are developed to support early drug discovery do not require regulated method validation. However, method qualification is advisable to ensure consistent and reproducible data. Once an assay is needed to support GLP-compliant (pre)clinical studies (e.g., safety, PK and biological equivalence), the method should be validated prior to the analysis of study samples [1]. The challenge is to fit flow cytometry into the current regulatory landscape for ligand-binding assay/ immunoassay method validation to meet FDA and EMA expectations.

Flow cytometry is most often used to detect specific cell markers in and on cells. Validating flow cytometric methods is challenging due to the inherent biological variability of cells, the limited stability of samples, the requirements for cytometer set-up and the complexity of the data output and interpretation of results [2-4].

Although there are no official regulatory guidance documents addressing the requirements for the validation of flow cytometric methods intended for use in drug development or for clinical testing, in the past few years, key stakeholders have published recommendation papers [3,5]. In 2011, members of the American Association of Pharmaceutical Scientists, Flow Cytometry Action Programming Committee published a paper describing the application and validation of flow cytometric methods in drug development [3]. In 2013, the International Council for Standardization of Hematology and
Barry van der Strate ${ }^{\star, 1}$, Robin Longdin ${ }^{2}$, Marie Geerlings', Nora Bachmayer ${ }^{3}$, Maria Cavallin ${ }^{4}$, Virginia Litwin ${ }^{5}$, Minesh Patel ${ }^{6}$, Wilfried Passe-Coutrin ${ }^{7}$, Corinna Schoelch $^{8}$, Arjen Companjen ${ }^{3}$ \& Marianne Scheel Fjording ${ }^{9}$ 'PRA Health Sciences, Amerikaweg 18, 9407 TK, Assen, The Netherlands 2LGC Ltd, Fordham, Cambridgeshire, UK ${ }^{3}$ Janssen ID\&V, Leiden, The Netherlands ${ }^{4}$ AstraZeneca, Macclesfield, UK ${ }^{5}$ Covance Central Laboratory Services, Indianapolis, IN 46214, USA ${ }^{6}$ Eurofins Pharma Bioanalysis Services, Abingdon, UK

${ }^{7}$ Sanofi, Alfortville, France ${ }^{8}$ Boehringer Ingelheim, Biberach an der Riss, Germany

${ }^{9}$ Novo Nordisk A/S, Måløv, Denmark

*Author for correspondence:

Tel.: +32 479910132

publications@europeanbioanalysisforum. eu

newlands press part of 
International Clinical Cytometry Society dedicated an entire issue of the Clinical Cytometry Journal to this topic [5-10].

In this manuscript, the European Bioanalysis Forum presents additional practice guidance on the use of flow cytometry in a regulated environment and addresses areas not covered in the previous publications. These areas are divided into two parts: those relating to the laboratory infrastructure and those relating to the analytical method. The concepts presented herein are based on the consensus of discussions in the European Bioanalysis Forum Topic Team 32.

\section{The laboratory infrastructure}

This section covers the creation of a regulatory-compliant environment that enables and supports the generation of valid data. This includes laboratory documentations such as standard operating procedures, the training of personnel and instrument set-up and maintenance. Infrastructure considerations specific to flow cytometry laboratories include the instrument optical alignment, assay-specific instrument standardization, interinstrument correlation and compensation, assay process controls, internal controls (positive and negative) and critical reagent (antibody) lot monitoring.

\section{Instrumentation}

In regulated bioanalysis, validation of the instrument is required to demonstrate fitness for the intended purpose and the generation of reliable and reproducible data over time.

The unique challenges of validating flow cytometer systems stem from the lack of accuracy standards (such as quality controls [QCs] with nominal concentration values used in quantitative methods such as ELISA), the customizable nature of the data acquisition and analysis templates and the fact that the dataset will encounter multiple software systems and databases. The instrument and all data processing software need to be validated [4]. Previous publications have described solutions to the challenges of validating flow cytometer systems and suggestions for lifecycle management of the system from installation to decommission $[4,11]$.

Instrument set-up, maintenance \& performance It is critical that the laboratory has a robust process for daily instrument set-up and calibration. It is a requirement to perform the manufacturer's recommended daily set-up and daily monitoring processes common to most instruments but additional monitoring must also be conducted in order to ensure that the instrument is performing consistently [12]. The optical alignment, fluidics, laser power and PMT voltages must be monitored.
Commercially available hard-dyed microspheres with fluorescence intensity values equivalent to a number of reference fluorophores are commonly used for optimizing and tracking the cytometer performance. Because these microspheres can be used at multiple wavelengths, they can monitor all lasers and detectors. A baseline is set for each detector, from which the everyday performance can be traced. This is a convenient way to calibrate and track the day-to-day performance of the flow cytometer or to ensure crossinstrument standardization [9,12]. Practical solutions to accomplish standardization have been described elsewhere [13,14].

\section{Critical reagents}

As for all equipment, each laboratory should ensure that routine maintenance is performed as appropriate and documented for all equipment. Although every reagent is critical to generate valid results, the term 'critical reagent(s)' is defined as the essential components that are used in ligand-binding assays, whose particular characteristics influence assay performance [15]. The reagents that contribute the most to assay variability are assigned as critical. Reagents that are used for cell treatment during sample preparation such as fixatives, permeabilization and lyzing solutions are not considered critical, because these reagents do not contribute to the signal readout $[9,16]$. However, the effect of introducing new batches of these solutions (during method development, validation and bioanalysis) should always be evaluated.

All critical reagents should be accompanied by a certificate of analysis and/or material data sheet to verify the source and quality of the reagent and storage and usage records [9]. All protein-derived reagents (antibodies and labeled proteins), whether conjugated or unconjugated, contribute to signal readout and should be considered critical.

For flow cytometry, most protein-derived reagents will exhibit lot-to-lot differences due to variation in conjugation and staining intensities. This can result in potential shifts or trends in reagent performance. Managing variability of fluorescent-labeled tandem dyes within lots may already be challenging, but certainly lot-to-lot variability should be investigated to compensate for dye degradation.

It is most favorable to obtain sufficient protein reagent of the same lot number to perform a complete study, preferably the same lot as used in the method validation [17]. To exclude fluorescent output variability due to antibody lot-to-lot variation, a comparison run must be performed to compare the new lot to the current lot. The current lot and new lot have to match in signal output [2]. Because of limited unknown sam- 
ple stability, good critical reagent stock keeping and planning is important.

\section{Raw data: definition}

Raw data that are properly defined, controlled and stored form the bedrock of regulatory compliance. With increasing complexity and power of automated electronic data acquisition systems, the definition of raw data becomes ever more important and complex accordingly.

The Organization for Economic Co-operation and Development defines raw data as all original test facility records and documentation, or verified copies thereof, which are the result of the original observations and activities in a study [18].

The following recommendations for defining and storing raw flow cytometry data are based on this definition, as well as the general rule that raw data can only ever be created once. The aim throughout is to ensure that regulatory authorities can trace any result back to the moment the raw data were acquired.

Raw data should, therefore, be defined as a list mode binary data file written by the cytometer software during data acquisition. Data acquisition is the recording of events with their corresponding detector signals. Raw data files should be available for all acquisitions and re-acquisitions. Such list-mode files are large, typically coded and not human readable, so paper copies of the file are not practical and do not allow full and proper study reconstruction. The electronic list mode file must therefore be maintained in a validated and secure storage environment with robust back-up procedures. The actual transfer of raw data after acquisition from the flow cytometer to the secure storage environment has to be monitored and validated.

\section{Raw data: storage}

It is recommended that data are written to .fcs files during acquisition where possible and that the FCS file is defined as raw data accordingly. Subsequent data processing performed either using the same software as that used for acquisition or another third-party software package is typically saved as a proprietary file type, separately from the FCS file from which the raw data were sourced. This approach makes managing and securing raw data files simpler since the raw data cannot be over-written after processing (Figure 1).

In addition to the raw data, processed data are an important part of an overall package of data required to allow the reconstruction of data. Processed data should also be well defined, controlled and stored, whether in an electronic form, such as PDF files, or as a printed hard-copy record. It is important to note that while the raw data can only ever be created once during data acquisition, processed data may be created a number of times from the same raw data, whether that is part of a reiterative process or as a result of technical error.

\section{The analytical method}

This section covers the development and validation of regulatory-compliant methods, and includes the establishment of assay controls, gating rules, sample collection protocols and characterizing sample stability.

\section{Sample collection}

Flow cytometry analyses can be performed using various different types of matrices. Differences in matrices and the assay end point will determine the structure of the method development and validation process. It is recommended, as a minimum, that during the preanalytical phase of a study, the sample type, volume required, frequency of the target population, choice of anticoagulant, sample handling, sample storage conditions and sample stability are considered. Relating these factors to the assays that will perform analysis is important to determine whether methods are feasible [16].

The (pre)clinical samples should be of the highest quality possible and the handling of samples and treatment should be considered. Factors such as the site of bleed, number of collections, trauma caused during the collection, postcollection storage, effect of processing and shipment of samples should be considered and assessed where appropriate [16].

Such considerations may form part of a sample collection guide to detail the correct procedure for sample processing, either at the clinical/preclinical sites, central laboratory or the analytical laboratory. Defining the sample collection guide as early as possible in the drug-development process is highly advantageous to the efficient planning and execution of preclinical and clinical studies. Tracking of samples, collection times, processing times, storage, operators and transport should be monitored during the study phase up to the time of sample analysis.

\section{Anticoagulants, preservation tubes \& postsample collection stability}

All biological materials, in particular living cells, begin to deteriorate once removed from the body. This fact brings the biggest challenge for flow cytometric analysis. Therefore, before method development starts, it is essential to have an understanding of what matrix has to be used, which cells are going to be analyzed and the time frame to ship samples from the clinical or preclinical specimen collection site to the testing laboratory.

The choice of the anticoagulant will influence the assay performance and stability of the specimen [19]. 


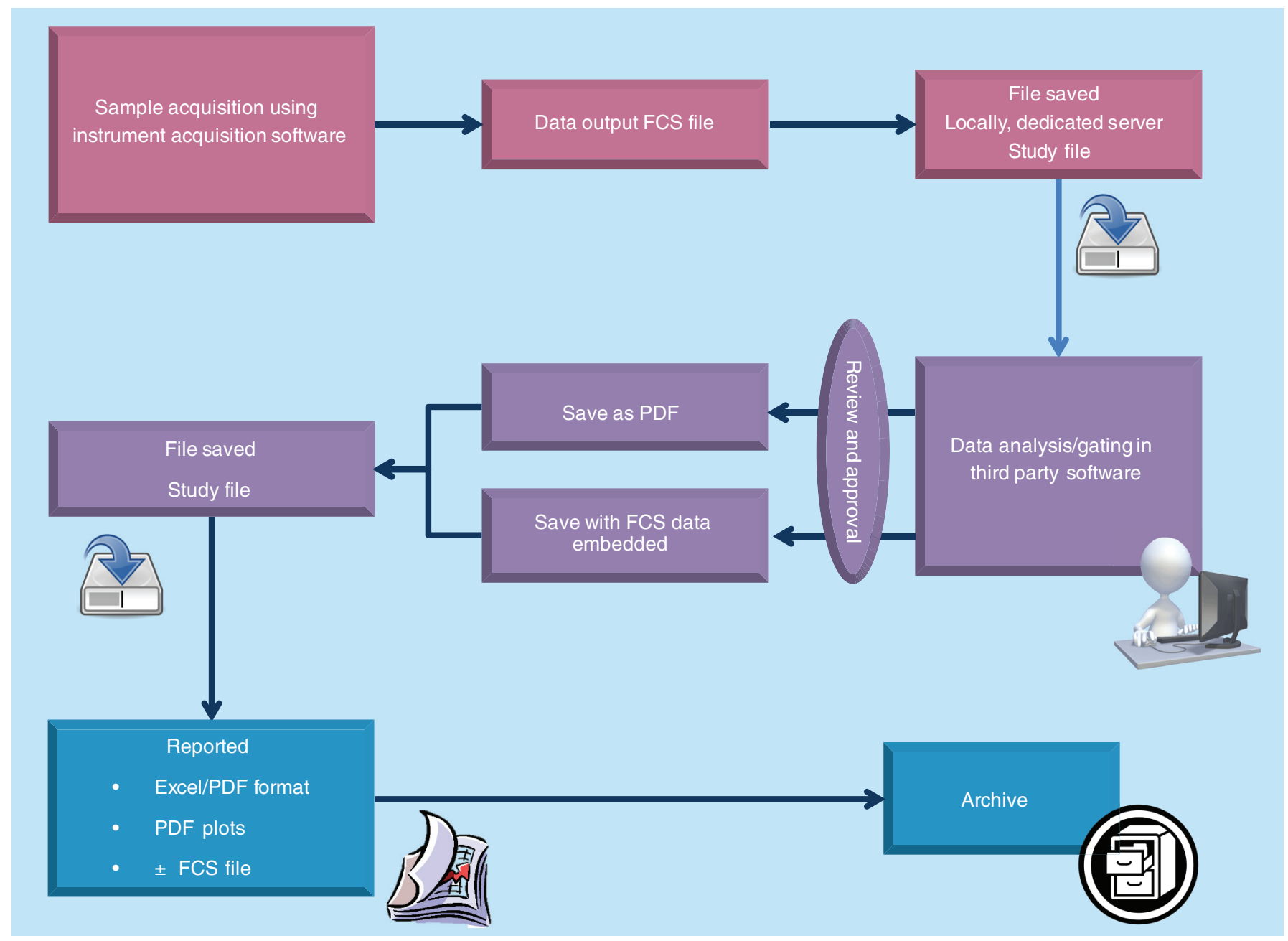

Figure 1. The flow of data from sample acquisition to the archive.

For instance, EDTA binds calcium and heparin can activate certain cells $[8,20,21]$. The optimal anticoagulant must be determined during the method development phase $[2,16,19]$. Preservation tubes can be used if the sample stability is not long enough to ship a sample from the clinical site to the laboratory in a standard blood collection tube [22]. Several FDA-approved whole blood stabilization products that improve stability of cell surface markers are available on the market (such as TransFix ${ }^{\circledR}$ [Buckingham, UK], Cyto-Chex $\mathrm{BCT}^{\circledR}[\mathrm{NE}, \mathrm{USA}]$ and CellSave ${ }^{\circledR}[\mathrm{NJ}, \mathrm{USA}]$, among others) [23,24]. Importantly, these products can only be used if cell stimulation is not required, because these stabilization products fix the whole blood and can alter the antigen expression levels or mask-specific epitopes.

Sometimes peripheral blood mononuclear cells (PBMCs) are more suitable than whole blood if the cells of interest are very rare in whole blood and the population of such cells must therefore be enriched. When it is not feasible to transport whole blood samples within the sample stability limitations, or when functional assays are performed on the cells of interest, isolation of PBMCs may be required. PBMCs can be isolated using density gradient centrifugation. PBMCs can be isolated and frozen directly at clinical sites; alternatively, a central laboratory can offer a solution [19].

\section{Method development}

Prior to method development, the assay requirements must be defined (Box 1), and it should be clear for what purpose the assay will be used. Are they obtained flow cytometry data of an exploratory nature, or are they used as a pivotal PD assay to demonstrate efficacy? Whereas for exploratory data method validation is not required, it is recommended that the assay is at least characterized during method development and is scientifically robust [3].

For the method development a reagent panel is designed (Box 1) [8,9,25-27]. The selection of reagents will influence the robustness of assay responses and assay sensitivity. 
The right sample collection matrix is chosen, and the optimal antibody concentrations, washing, lysing and fixation conditions are established. While setting up the assay, appropriate (negative or positive) controls should be defined. If the method has been set up, it is recommended to assess the most important validation parameters such as precision and stabilities (whole blood stability and stained cell stability) prior to start of the actual validation of the method, to prevent surprises during validation. It is recommended to prepare a summary document of the assay development strategy objectives, the intended use, readout of the assay, frequency (i.e., percentage) and/or grade of positivity (i.e., fluorescence) of cells.

\section{Specificity}

The standard definition of specificity is the ability of a measurement procedure to measure solely the analyte. In a regulated environment, specificity is addressed by establishing cross-reactivity of the reagents. This point is particularly important for enzyme immunoassays and related methods where the antibody reagent might cross-react with a similar protein or recognize the wrong form of the analyte, such as the precursor or a degradation product.

Although this is a validation parameter, specificity is already addressed during method development. The selection of antigens to define a given cellular subset, the $\mathrm{mAb}$ clone selection, the fluorochrome$\mathrm{mAb}$ pairing, the $\mathrm{mAb}$ titration, buffer selection, wash sequences and gating strategy, all influence the ability to specifically measure the population of interest.

\section{Gating strategy}

During method development a gating strategy is defined and standardized. The gating strategy should contain the minimal or maximal gating boundaries to minimize data variability $[28,29]$. It is important that the gate setting is performed consistently during the course of the bioanalytical study to minimize data variability and to prevent conscious or unconscious data manipulation.

The raw data acquired from the flow cytometer needs to be processed to obtain data for independent evaluation [30]. To ensure that the data can be evaluated, list mode data files, preferably in '.fcs' format, are processed by using software from the vendor of the flow cytometer or by using software from a third party, for example, FlowJo $^{\mathrm{TM}}$ (Ashland, OR, USA), FACSDiva ${ }^{\mathrm{TM}}$ (Becton Dickinson, SJ, USA), Infinicyt ${ }^{\mathrm{TM}}$ (Becton Dickinson), CellQuest Pro ${ }^{\mathrm{TM}}$ (Becton Dickinson), Winlist ${ }^{\mathrm{TM}}$ (Verity Software House, ME, USA), FCS Express ${ }^{\mathrm{TM}}$ (De Novo Software, CA, USA), Kaluza, Guava ${ }^{\mathrm{TM}}$ (Beckman Coulter, IN, USA) and many others [30,31].
Gating can be performed in a univariate manner to produce a histogram. Alternatively, bivariate (to produce dot/contour plots) or trivariate data can be produced. Both manual and automatic gating is possible [31,32]. When applying automatic gating, the chance of data variability are considered low, and should be used when knowing the proper cell populations.

With manual gating, particular precaution must be taken as manual gating can only be done accurately when enough knowledge about biological processes and sufficient experience with flow cytometric gating are available. Still, it is difficult to have uniform gating, since the judgment of what is an appropriate gate can differ from person to person. Establishing standardized, controlled analysis templates can reduce variability [33].

In some cases, it is not possible to apply the predefined gating strategy, such as instances when matrices of diseased subjects cause gating challenges. For example, patients with leukemia can have abnormally high cell numbers or other diseases can cause increased adhesiveness of cells. When bioanalytical samples have issues and generate abnormal plots, gating can become challenging. Because some samples have limited sample stability, it is not always possible to re-analyze these samples. If these cytometry samples are very precious, and analysis cannot be repeated, a common sense gating approach can be applied. Deviation of the predefined gating strategy must be documented with reasons. The data must be flagged after deviation of the gating strategy. Different strategies can be used to identify positive and negative populations as a control for the gating performed during the study.

\section{Box 1. Method development.}

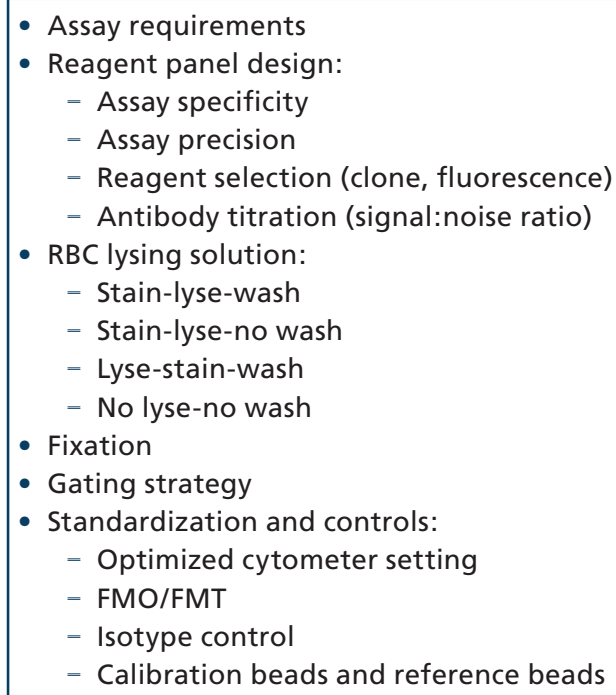

FMO: Fluorescence Minus One; FMT: Fluorescence Minus Two; RBC: Red blood cells. 


\section{Gating controls}

Before discussing the value of Fluorescence Minus One (FMO), isotype controls and isoclonic controls, panel design and gating strategy must be reviewed. An appropriate reagent selection and gating strategy must be established during method development to generate the highest degree of specificity. This ensures that the events detected in the final gate for the reportable result are real, and not due to compensation issues, nonspecific antibody binding, a contaminating cell type, air bubbles or dead or clumped cells.

The use of a time plot allows for the elimination of spurious events, and additionally doublet discrimination plots should be included. Next, a series of sequential gates can be applied to specifically identify a particular cellular type. For example CD45, CD3, CD14, CD56 and CD19 might be used to identify CD19 B cells in peripheral whole blood. First, lymphocytes would be gated from the other leukocytes based on high CD 45 and low SSC properties. Then CD3 T cells, CD56 natural killer cells and any containing CD14 monocytes can be negatively selected. Final CD19-positive B cells can be positively gated with high specificity. The selection of antigen-positive events versus antigen-negative events is straightforward with CD45, CD3, CD14, CD56 and CD19, as the positive events are well separated from the negative events. A common error in establishing the lymphocyte gate is the inclusion of some monocytes. Adding CD14 in a downstream gate will correct for any errors made in establishing the lymphocyte gate.

If the goal of the assay was to identify activated B cells based on CD69 expression, several approaches could be applied to establish the criteria for fluorescence positivity of the fluorochrome conjugated to the anti-CD19 mAb. The current best practice is the use of an FMO control where all reagents except CD69 are included in the gating control tube. In this approach, the positive:negative boundary established on CD19 B cells accounts for the spread in this population resulting from postcompensation spread and autofluorescence distributions [34].

Conceptually, the use of an isotype control would be the preferred method, but isotype controls have many shortcomings and their application as gating controls has not been considered the best practice by the flow cytometry community for more than a decade [35]. An isotype control is an immunoglobulin of the same species, isotype and fluorochrome conjugation as the antigen-specific fluorochrome-conjugated reagent, but with an 'irrelevant' specificity. The theory is that any nonspecific binding of the antibody or fluorochrome to the cell would be detected with the isotype. There are several reasons why, in practice, this is not the case, and numerous publications have focused on this topic [35]. The primary reason why isotype controls are not the optimal gating control is that they do not contain the same fluorescence-to-protein ratio as the antigen-specific fluorochrome conjugated reagent.

Another way to establish the antigen-specific fluorochrome signal is the addition of an excess of unlabeled antigen-specific antibody, or an isoclonic control [35]. While valid, this approach will add additional expense to an assay and may result in unanticipated issues in polychromatic assays. In a research setting, or in the assay development phase, isoclonic controls would have value in establishing assay specificity.

Internal negative controls can also be used as gating controls. In this approach, a subset of cells that is not expressing the antigen-of-interest would be used as the negative control. This approach is only practical when both cell populations have similar autofluorescence properties. This approach presents a challenge in complex immunophenotyping where many of the antigens-of-interest are shared among the major lymphocytes subsets, so that an internal negative control is not actually available. The use of internal negative controls is highly valuable in the field of hematopathology, where both normal and abnormal cells are present in the sample and where the abnormal cells express antigens that are not found on the normal cell.

In summary, the ideal gating control depends on the intended use of the assay and the regulatory environment in which it is conducted. That said, by and large, the FMO approach is preferred for complex immunophenotyping.

\section{Use of QCs}

There are no absolutes in flow cytometry. The power of this technology stems from the inherent flexibility resulting in a vast number of potential applications (e.g., immunophenotyping, intracellular signaling, nucleic acid detection and functional activity). Consideration to the assay type, regulatory environment and intended use of the data must be taken into account when selecting the optimal QC material. In any laboratory, regulated or nonregulated, the inclusion of QC samples in every analytical run is advantageous, but when considering longitudinal data, or multicenter data, QC samples are critical. Even though in diagnostic clinical laboratories the use of internal cellular QCs is an accepted practice particularly in hematopathology, for assay supporting drug development and clinical trial testing the inclusion of external QCs is the best practice.

As with many aspects of flow cytometry a perfect solution for QC material does not exist. Ideally, the QC material will be both similar to the specimen 
matrix and have a long shelf life. The goal of a QC is to ensure that all of the reagents are functioning properly and to monitor trending in assay performance and not to monitor the flow cytometer performance.

Flow cytometric methods approved for in vitro diagnostic use will include qualified QC material, but even these QCs are imperfect. Whole blood methods for lymphocyte immunophenotyping of $\mathrm{T}$ cells, CD4 T cells, CD8 T cells, natural killer cells and $\mathrm{B}$ cells include preserved material similar to whole blood. This material offers the advantage of controlling for the erythrocyte lysis steps of methods performed in whole blood. Although target ranges are provided, the ranges are extremely broad and thus of little value for accuracy assessment. Each laboratory needs to establish its own range to be used for assay acceptance criteria. The major disadvantage of this material is that the low and normal QC levels are calibrated to the number of CD4 T cells. Other cell types may have the same number of cells in both the low and normal QC. The shelf life of these products is limited and is depending on the manufacturer. Thus, a longitudinal study will require the use of multiple lots, where each new lot of material is not necessarily calibrated to the former lot, resulting in the change of target values.

For whole blood immunophenotyping methods, the commercially available 'preserved' whole blood material can serve as a QC. In addition to the disadvantages encounter with in vitro diagnostic assays, some cell subsets or rare events may not be detectable in the QC material. It is critical that every antibody used in the assay be monitored. In cases where a particular reportable result is not resolved well in the QC material, an alternative cell type expressing that antigen should be monitored. Alternatively, the performance of the staining antibody could be monitored using antigen binding beads. This approach will only monitor the fluorescence integrity of the antibody and not the antigen binding aspect of the antibody, but most issues with the reagents will be related to degradation of the fluorochrome conjugates and not the antibody molecule.

An alternative for commercially available QCs is to prepare them in-house. For instance, a batch of lyophilized cells enriched for the particular cell type. Lyophilized cells will have a long shelf-life but will not allow for controlling the RBC lysis steps, if required. A combination of preserved whole blood and lyophilized QC would ensure that all steps of the assay and each reportable cell type are accounted for in at least one assay QC. Additionally, it is possible to use isolated PMBCs or cell lines as QCs to monitor assay performance and for run acceptance purposes.

\section{Method validation}

Method validation is the confirmation that the performance characteristics of an assay are suitable and reliable for its intended use. In order to design an appropriate validation plan, it is critical to fully understand the data output. Most often flow cytometry data will be categorized as quasi-quantitative, given that the results are numeric and are proportional to the measured test sample characteristic, but they are not derived from a reference standard [36].

Because of the different applications where flow cytometry can be used for (e.g., immunophenotyping, PK, PD, biomarker and functional assays) method validation parameters will depend on the intended use of data. For example, assays for compound screening, exploratory PD biomarkers, surrogate end point and companion diagnostic will have increasing requirements for the method validation [37].

In the past several years, recommendation papers have been published that describe the validation of instrument and flow cytometry methods $[3-5,9,11,38,39]$. After successful completion of method development, the following aspects should be incorporated into the formal validation of the method.

\section{Validation parameters \\ Precision}

Any bioanalytical assay should yield reproducible results and precision. Different aspects of precision should be addressed during validation of a flow cytometry method. The variation between separate runs (intra-assay precision) and within a run should be assessed. Next to precision of the assay, the biological variation for a specific assay should be assessed. Care should be taken to investigate both the intra- and intersubject variability.

Another source of variability in assay results can be introduced by sample processing of different technicians (interoperator reproducibility). Additional variability can occur during data processing (gating) by different analysts. Therefore, interanalyst data processing reproducibility should be assessed.

\section{Sensitivity}

The standard definition of sensitivity is the lowest reportable result. Sensitivity includes the limit of detection or the limit of blank as well as the lower limit of quantitation. For flow cytometric methods, strategies for establishing the limit of detection/limit of blank and the lower limit of quantitation depend on the assay format and final reportable results for the method $[3,5,40]$. When the assay read-out is fluorescence intensity, the maximum fluorescence intensity of a positive population and the maximum separation between 
positive and negative populations greatly influences assay sensitivity [39]. Sensitivity assessment should determine both the maximum fluorescence intensity of a positive population and the maximum separation between positive and negative populations [39].

\section{Robustness}

The robustness of an assay describes the capacity of a method to remain unaffected by small but deliberate changes in method parameters and provides an indication of its reliability during normal run conditions. It is recommended to test several detection antibody lot numbers, as well as different analysts. The assumption that the gating differences between two persons are within the variation of the assay can be incorrect and should also be incorporated into the validation $[2,41]$.

\section{Accuracy}

In most flow cytometry assays, it is not possible to determine accuracy, for example, when comparing changes in specific cell-surface markers in an individual, because true cellular standard reference materials with appropriate biological matrices are not available. In some cases it may be possible to include internal or commercially available QC samples $[3,5,9,40,42]$.

\section{Stability}

Stability testing should reflect the same conditions that samples would be subjected to during the trial and during analysis. The stability of an analyte in a given matrix under specific conditions should be determined to identify analyte stability limits, including time from sample collection to the time of analysis, storage conditions and freeze-thaw cycles $[5,8,19]$. These assessments should be performed within the same sample collection tube and should mirror the anticoagulants, preservatives and materials used by clinicians at the trial sites.

Results from such assessments should be compared against the baseline sample to ensure the stability data are within suitable acceptance limits.

\section{Long- \& short-term frozen stabilities}

Determination of short-term and long-term stability (depending on sample type: this may vary from hours to days, respectively for cells, or to multiple weeks/ months frozen stability) may impose time constraints and delay the start of the sample analysis study. It may be necessary to validate the stability by an instudy validation. Such assessments would be dependent upon the regulatory environment of the study. In general, these validations should be performed months before the start of the (pre)clinical trial if possible and should mimic the processes at the trial site as much as possible for comparative analysis to allow for identification and troubleshooting of poorly processed samples.

When using frozen PBMC to prolong the period of collection and acquisition, the effect of cryopreservation or stabilization on marker expression (activation) and the effect of freezing on the cell viability (cell death) must be evaluated. A selective loss of cells after freeze-thaw or a detrimental effect of a freeze-thaw cycle to the samples may occur $[2,16,39]$.

\section{Postsample processing stability}

Sometimes it is not possible to analyze samples on a flow cytometer immediately after staining for several reasons (e.g., instrument failure and extended run times). Therefore, the postsample processing stability should be determined.

After the sample processing is completed, samples can be suspended in a buffer containing phosphatebuffered saline with a protein, followed by a treatment with a fixative to withstand delays in acquisition. However, caution should be taken in account when fixing cells as some fluorophores or markers cannot be fixed. In most case, the fixative of choice is either $1-3 \%$ formaldehyde or paraformaldehyde.

\section{Run acceptance criteria}

Batch and sample acceptance criteria are predefined for each method and based on method validation data and documented in either the analytical plan. Like any methodology run acceptance criteria are primarily based on the QC samples being within the accepted range. Exact criteria are evaluated on a case-by-case basis. With flow cytometric methods, a visual inspection of the gated data, either automatically or manually gated, must be conducted to ensure that all aspects of the staining and acquisition are performing as expected. This inspection should include verification that correction of compensation was applied. Further guidance on method validation and acceptance criteria are available in 'Recommendations for the validation of flow cytometric testing during drug development: II assays' by O'Hara et al. [3].

\section{Method validation \& bioanalytical report}

Summaries of method development, method validation reports or bioanalytical reports should adhere to a minimum of requirements that are essential for reconstruction of the conducted study. Moreover, proper validation and bioanalytical reports facilitate successful transfer of analytical methods between different laboratories [10].

It is recommended that reports that are generated in a regulated environment should at least contain the items (Table 1). 
Table 1. Minimal Requirements for validation and analytical reports.

\begin{tabular}{|c|c|c|}
\hline Headings & Validation report & Analytical report \\
\hline Aim of study & $\begin{array}{l}\text { Description of assay and the intended use of data } \\
\text { (exploratory versus decision-making) }\end{array}$ & $\begin{array}{l}\text { - Description of what is measured and what } \\
\text { the intended use of data (exploratory vs } \\
\text { confirmatory) is } \\
\text { - Brief conclusion }\end{array}$ \\
\hline Assay procedure & $\begin{array}{l}\text { - Method description (stepwise) } \\
\text { - antibody panel } \\
\text { - Cytometer } \\
\text { - Reagents (including donor batch, catalogue } \\
\text { number for antibody, batch number) } \\
\text { - Calibration beads/MESF beads }\end{array}$ & $\begin{array}{l}\text { - Flow cytometry apparatus/instrument } \\
\text { - Analytical procedure/method description } \\
\text { (stepwise) } \\
\text { - Critical reagents (incl. batch no. calibration } \\
\text { beads, MESF beads and antibodies) } \\
\text { - Antibody panel }\end{array}$ \\
\hline Acceptance criteria & Short description & $\begin{array}{l}\text { Short description of the acceptance criteria } \\
\text { predefined in the validation report }\end{array}$ \\
\hline Validation parameters & $\begin{array}{l}\text { - 1. Precision } \\
\text { - Intra-assay precision } \\
\text { - Interassay precision } \\
\text { - 2. Biological variability } \\
\text { - Intrasubject variability } \\
\text { - Intersubject variability (if possible in disease } \\
\quad \text { state; depends on intended use of data) } \\
\text { - 3. Stability } \\
\text { = Postcollection stability } \\
\text { - Postfixation stability } \\
\text { - 4. Sensitivity } \\
=\text { Blood volume for sample staining } \\
\text { - Number of events for sample acquisition }\end{array}$ & NA \\
\hline Samples & $\begin{array}{l}\text { Description of how the samples should be taken } \\
\text { incl. matrix and storage }\end{array}$ & $\begin{array}{l}\text { - Sample receipt (number expected, number } \\
\text { received and number analyzed) and storage } \\
\text { - Sample processing } \\
\text { - Run overview including acceptance/rejection } \\
\text { status }\end{array}$ \\
\hline $\begin{array}{l}\text { Data processing and } \\
\text { storage }\end{array}$ & & $\begin{array}{l}\text { - Raw data acquisition and storage } \\
\text { - Analysis of raw data }\end{array}$ \\
\hline $\begin{array}{l}\text { Gating and analysis } \\
\text { procedures }\end{array}$ & & Incl. gating strategy and reportable \\
\hline Results & $\begin{array}{l}\text { Incl. summary tables and if acceptance criteria } \\
\text { were met }\end{array}$ & $\begin{array}{l}\text { Incl. summary tables and if acceptance criteria } \\
\text { were met }\end{array}$ \\
\hline Conclusion & Incl. reportable and how gating should be set & $\begin{array}{l}\text { Description of assay performance and if data } \\
\text { are valid }\end{array}$ \\
\hline Deviation & $\begin{array}{l}\text { Description of deviations and impact on study } \\
\text { results }\end{array}$ & $\begin{array}{l}\text { Description of deviations and impact on study } \\
\text { results }\end{array}$ \\
\hline \multicolumn{3}{|l|}{ Reference list } \\
\hline \multicolumn{3}{|l|}{ Abbreviations } \\
\hline Appendix & $\begin{array}{l}\text { - Raw data tables (incl. exemplary plots/histograms) } \\
\text { - Gating strategy } \\
\text { - Analytical SOP }\end{array}$ & $\begin{array}{l}\text { - Data result tables (incl. exemplary plots/ } \\
\text { histograms) } \\
\text { - Calibration beads } \\
\text { - Analytical SOP }\end{array}$ \\
\hline
\end{tabular}




\section{Conclusion}

In this article we propose best practices based on the experience from the team members recommendations for performing flow cytometry studies (validation and bioanalysis), which are based on a summary of current publications and the authors' specific experiences. Assays that are developed to support early R\&D discovery have different needs compared with an assay that reaches the need to support regulatory compliant preclinical and clinical studies, for safety, PK and biological equivalence. In this aspect, flow cytometry methods are not different from any other methods and requirements, that is used for the support of (pre) clinical studies in a regulated environment.

Next to the standard parameters that need to be addressed for other immunoassays such as an ELISA, performing flow cytometry in regulated environment requires additional attention for some specific (flowrelated) items. Since in flow cytometry, accuracy cannot be determined, additional attention should be given to precision of the flow method, precision between multiple operators in method performance, but also in data processing. (in other words: make sure that multiple operators end up with the same end results when processing samples and rocessing acquired data). The use of QCs for run acceptance control and method performance monitoring is essential.

Finally, some last words on the additional challenges of multisite studies. When flow cytometric assays are performed on several cytometers at one facility or in multiple locations, it is essential to ensure that results are comparable. Therefore, the standardization of cytometer settings across the different cytometers and continuous performance monitoring (of cytometer and assay) become paramount. It is recognized that sources of variation can be present at the different phases of analysis: pre-analytical (sample handling), analytical (sample analysis) and postanalytical (data handling), which can influence or compromise the comparability of the data. In multisite flow cytometry analysis, the multicytometer or multisite setting should be taken into account and incorporated into the method validation plans.

\section{References}

1 Findlay JW, Smith WC, Lee JW et al. Validation of immunoassays for bioanalysis: a pharmaceutical industry perspective. J. Pharm. Biomed. Anal. 21(6), 1249-1273 (2000).

2 Wu DY, Patti-Diaz L, Hill CG. Development and validation of flow cytometry methods for pharmacodynamic clinical biomarkers. Bioanalysis 2(9), 1617-1626 (2010).

3 O'Hara DM, Xu Y, Liang Z, Reddy MP, Wu DY, Litwin V. Recommendations for the validation of flow cytometric

\section{Future perspective}

Flow cytometry is increasing in popularity for its versatile applicability in the support of (pre)clinical trials. This popularity is expected to further increase over the years, as the development of new flow cytometry platforms will further enhance the possibilities. Newer flow cytometry platforms have the capabilities to detect up to 20 different colors.

This increase in detection capabilities will enable scientists to dive deeper into a specific cell population of interest by including multiple markers to distinguish cell types and/or to investigate pharmacological activity. Alternatively, multiple cell populations can be analyzed from a single tube, rather than having to use multiple tubes. This development will certainly result in lower blood volumes that have to be used during a (pre) clinical trial.

Another exciting development is the use of CyTOF (combination of flow cytometry and TOF), a combination of time-of-flight analysis and cytometry. This platform will enable the possibilities even more, as it is capable of analyzing even more markers at the same time (up to 100).

A lot of the newer platforms are currently used in a research setting, but we may expect these newer platforms to enter the regulated bioanalytical arena where they can contribute to the support of (pre)clinical trials. Finally, the versatility of flow cytometry will result in increased use of flow cytometry in different 'disciplines' such as pharmacokinetics (and receptor occupancy) and determination of immunogenicity and neutralizing antibody activity.

\section{Financial \& competing interests disclosure}

The authors have no relevant affiliations or financial involvement with any organization or entity with a financial interest in or financial conflict with the subject matter or materials discussed in the manuscript. This includes employment, consultancies, honoraria, stock ownership or options, expert testimony, grants or patents received or pending, or royalties.

No writing assistance was utilized in the production of this manuscript.

testing during drug development: II assays. J. Immunol. Methods 363(2), 120-134 (2011).

4 Green CL, Brown L, Stewart JJ, Xu Y, Litwin V, McCloskey TW. Recommendations for the validation of flow cytometric testing during drug development: I instrumentation. J. Immunol. Methods 363(2), 104-119 (2011).

5 Wood B, Jevremovic D, Béné MC et al. Validation of cellbased fluorescence assays: practice guidelines from the ICSH and ICCS - part V - assay performance criteria. Cytometry $B$ Clin. Cytom. 84(5), 315-323 (2013). 
6 Béné MC, Marti GE. ICSH/ICCS practice guidelines special issue. Cytometry B Clin. Cytom. 84(5), 279-280 (2013).

7 Davis BH, Wood B, Oldaker T, Barnett D. Validation of cell-based fluorescence assays: practice guidelines from the ICSH and ICCS - part I - rationale and aims. Cytometry B Clin. Cytom. 84(5), 282-285 (2013).

8 Davis BH, Dasgupta A, Kussick S, Han JY, Estrellado A, Group IIW. Validation of cell-based fluorescence assays: practice guidelines from the ICSH and ICCS - part II - preanalytical issues. Cytometry B Clin. Cytom. 84(5), 286-290 (2013).

9 Tanqri S, Vall H, Kaplan D et al. Validation of cell-based fluorescence assays: practice guidelines from the ICSH and ICCS - part III - analytical issues. Cytometry B Clin. Cytom. 84(5), 291-308 (2013).

10 Barnett D, Louzao R, Gambell P et al. Validation of cellbased fluorescence assays: practice guidelines from the ICSH and ICCS - part IV - postanalytic considerations. Cytometry B Clin. Cytom. 84(5), 309-314 (2013).

11 Ferbas J, Schroeder MJ. Instrument validation for regulated studies. In: Flow Cytometry in Drug Discovery and Development. Litwin V, Marder P (Eds). John Wiley \& Sons Inc., NJ, USA, 267-277 (2010).

12 Perfetto SP, Ambrozak D, Nguyen R, Chattopadhyay PK, Roederer M. Quality assurance for polychromatic flow cytometry using a suite of calibration beads. Nat. Protoc. 7(12), 2067-2079 (2012).

13 Degheidy H, Abbasi F, Mostowski $\mathrm{H}$ et al. Consistent, multiinstrument single tube quantification of CD20 in antibody bound per cell based on CD 4 reference. Cytometry B Clin. Cytom. 90(2), 159-167 (2016).

14 Perfetto SP, Chattopadhyay PK, Wood J et al. Q and B values are critical measurements required for inter-instrument standardization and development of multicolor flow cytometry staining panels. Cytometry A 85(12), 1037-1048 (2014).

15 O'Hara DM, Theobald V, Egan AC et al. Ligand binding assays in the 21st century laboratory: recommendations for characterization and supply of critical reagents. AAPS J. 14(2), 316-328 (2012).

16 Cunliffe J, Derbyshire N, Keeler S, Coldwell R. An approach to the validation of flow cytometry methods. Pharm. Res. 26(12), 2551-2557 (2009).

17 Geist BJ, Egan AC, Yang TY, Dong Y, Shankar G. Characterization of critical reagents in ligand-binding assays: enabling robust bioanalytical methods and lifecycle management. Bioanalysis 5(2), 227-244 (2013).

18 Organisation for Economic Co-operation and Development. OECD series on principles of good laboratory practice and compliance monitoring. OECD Publishing, Paris, France (1998).

19 Brown L, Green CL, Jones N et al. Recommendations for the evaluation of specimen stability for flow cytometric testing during drug development. J. Immunol. Methods 418, 1-8 (2015).

20 Shalekoff S, Page-Shipp L, Tiemessen CT. Effects of anticoagulants and temperature on expression of activation markers CD11b and HLA-DR on human leukocytes. Clin. Diagn. Lab. Immunol. 5(5), 695-702 (1998).

21 Hernandez MR, Bozzo J, Tonda R, Galan AM, Ordinas A, Escolar G. Effect of anticoagulants on activation of polymorphonuclear leukocytes induced by shear stress. Int. J. Immunopathol. Pharmacol. 14(3), 139-144 (2001).

22 Pinto LA, Trivett MT, Wallace D et al. Fixation and cryopreservation of whole blood and isolated mononuclear cells: influence of different procedures on lymphocyte subset analysis by flow cytometry. Cytometry B Clin. Cytom. 63(1), 47-55 (2005).

23 Canonico B, Betti M, Luchetti F et al. Flow cytometric profiles, biomolecular and morphological aspects of transfixed leukocytes and red cells. Cytometry B Clin. Cytom. 78(4), 267-278 (2010).

24 Warrino DE, DeGennaro LJ, Hanson M, Swindells S, Pirruccello SJ, Ryan WL. Stabilization of white blood cells and immunologic markers for extended analysis using flow cytometry. J. Immunol. Methods 305(2), 107-119 (2005).

25 Wood B. 9-color and 10-color flow cytometry in the clinical laboratory. Arch. Pathol. Lab. Med. 130(5), 680-690 (2006).

26 McLaughlin BE, Baumgarth N, Bigos M et al. Nine-color flow cytometry for accurate measurement of $\mathrm{T}$ cell subsets and cytokine responses. Part I: panel design by an empiric approach. Cytometry A 73(5), 400-410 (2008).

27 McLaughlin BE, Baumgarth N, Bigos M et al. Nine-color flow cytometry for accurate measurement of $\mathrm{T}$ cell subsets and cytokine responses. Part II: panel performance across different instrument platforms. Cytometry A 73(5), 411-420 (2008).

28 Shapiro HM. Practical Flow Cytometry. John Wiley \& Sons, Inc. (2005).

29 Orfao A. Phenotypic analysis. Current Protocols in Cytometry. Wiley Online Library (2009).

30 Spidlen J, Moore W, Parks D et al. Data File Standard for Flow Cytometry, version FCS 3.1. Cytometry A 77(1), 97-100 (2010).

31 O’Neill K, Aghaeepour N, Spidlen J, Brinkman R. Flow cytometry bioinformatics. PLoS Comput. Biol. 9(12), e1003365 (2013).

32 Naumann U, Luta G, Wand MP. The curvHDR method for gating flow cytometry samples. BMC Bioinformatics 11,44 (2010).

33 Maecker HT, McCoy JP, Nussenblatt R. Standardizing immunophenotyping for the Human Immunology Project. Nat. Rev. Immunol. 12(3), 191-200 (2012).

34 Tung JW, Heydari K, Tirouvanziam R, Sahaf B, Parks DR, Herzenberg LA. Modern flow cytometry: a practical approach. Clin. Lab. Med. 27(3), 453-468, v (2007).

35 Hulspas R, O'Gorman MR, Wood BL, Gratama JW, Sutherland DR. Considerations for the control of background fluorescence in clinical flow cytometry. Cytometry B Clin. Cytom. 76(6), 355-364 (2009).

36 Lee JW, Weiner RS, Sailstad JM et al. Method validation and measurement of biomarkers in nonclinical and clinical samples in drug development: a conference report. Pharm. Res. 22(4), 499-511 (2005). 
37 Lee JW, Devanarayan V, Barrett YC et al. Fit-for-purpose method development and validation for successful biomarker measurement. Pharm. Res. 23(2), 312-328 (2006).

38 Cummings J, Raynaud F, Jones L, Sugar R, Dive C. Fitfor-purpose biomarker method validation for application in clinical trials of anticancer drugs. Br. J. Cancer 103(9), 1313-1317 (2010).

39 Hill C, Wu D, Ferbas J, Litwin V, Reddy M. Regulatory compliance and method validation. In: Flow Cytometry in Drug Discovery and Development. Litwin V, Marder P (Eds). John Wiley \& Sons, Inc., NJ, USA, 243-266 (2010).
40 Litwin V. CYTO 2014 tutorial "Understanding Analytical Method Validation as Applied to Flow Cytometry" (2014). http://isac-net.org/PDFS/df/df2b0b45-95e4-4221-

41 Maecker HT, McCoy JP, Amos M et al. A model for harmonizing flow cytometry in clinical trials. Nat. Immunol. 11(11), 975-978 (2010).

$42 \mathrm{Xu}$ Y, Richards S. Pharmacokinetics by flow cytometry: recommendations for development and validation of flow cytometric method for pharmacokinetic studies. In: Flow Cytometry in Drug Discovery and Development. Litwin V, Marder P (Eds). John Wiley \& Sons, Inc., NJ, USA (2010). 